\title{
Large-Eddy Simulation of the Flow Around a Ground Vehicle Body
}

\author{
Siniša Krajnović and Lars Davidson \\ Department of Thermo and Fluid Dynamics \\ Chalmers University of Technology \\ SE-412 96 Göteborg, Sweden
}

Copyright (C) 2001 Society of Automotive Engineers, Inc.

\begin{abstract}
Large Eddy Simulation of the the flow around buslike ground vehicle body is presented. Both the timeaveraged and instantaneous aspects of this flow are studied. Time-averaged velocity profiles are computed and compared with the experiments [1] and show good agreement. The separation length and the base pressure coefficient are presented. The predicted pumping process in the near wake occurs with a Strouhal number $S t=0.073$, compared with $S t=0.069$ in the experiment. Unsteady results at two points are presented and compared with the experiments. The coherent structures are studied and show good agreement with the experiments.
\end{abstract}

\section{INTRODUCTION}

Computations based on Reynolds-Averaged NavierStokes Equations (RANS) are common in industry today. In the 1990s new non-linear eddy viscosity turbulence models were developed that proved to be reasonably accurate and numerically stable ( see e.g. [2, 3]). Although they are very successful in predicting many parts of the flow around a vehicle, they are unable to predict unsteadiness in the wake region. The failure in predicting the base pressure is the major reason for the large discrepancy in drag prediction between experiments and RANS simulations. An additional problem in RANS simulations is obtaining a steady-state solution when a very fine grids are used [4]. There are also many transient phenomena such as vortex shedding and accumulation of dirt or water on the vehicle. These can only be predicted using a transient simulation such as Large Eddy Simulation (LES) or unsteady
RANS [5].

Early LES simulations were made of such flows as homogeneous turbulence, plane channel flow and mixing layers. More complex flows such as those around a surface-mounted cube $[6,7,8]$ and around a rectangular cylinder $[6,9,10]$ have recently been simulated using this technique. In their simulations, of the flow around a surface-mounted cube, Krajnović and Davidson [7] showed that it is possible to obtain accurate results at a computational cost of only $60 \mathrm{CPU}$ hours on an SGI R10000. The results of these simulations encourage us to go further with more complex geometry and higher Reynolds numbers towards the simulation of the flow around a vehicle.

There is growing interest in the automotive industry in exploring the possibilities of this new technique, especially at Volvo Car Corporation, which supports this project. Before we begin with Large Eddy Simulation of the flow around vehicle, it is worth noting that there are three large differences between this flow and the flow around a surface-mounted cube [7]. The separations in the flow around a surface-mounted cube are well defined by the sharp edges of the cube, whereas there is no such definition for the separation from the surface on the vehicle except at the rear end (see Fig. 1). A second difference between these two flows is the Reynolds number which, in the case of the cube, was 40000 based on the incoming bulk velocity and the cube height. The Reynolds number for the flow around the vehicle is $1-2$ orders of magnitude higher. Finally, the cube was mounted on the wall, and the absence of flow under the cube damped the vortex shedding and made the instantaneous wake shorter. In the case of the ve- 
hicle, which is lifted from the floor, the outlet boundary must be moved further downstream to prevent the unphysical damping of the instantaneous wake. The first two differences imply higher resolution requirements in the near wall region in the case of the vehicle while the longer downstream length means a larger computational domain. The increase in the spatial resolution leads inevitably to the increase in the time resolution owing to the stability requirements (CFL number). These requirements on the resolution make this kind of simulation extremely time consuming and computationally demanding. Even though computers are becoming faster, the wall-resolved LES of this flow is still unaffordible. The first grid point must be located at $y^{+}<2$ expressed in the wall units $y^{+}=\bar{u}_{\tau} y / \nu$. The resolution in the streamwise and the span-wise directions expressed in the wall units must be $\Delta x^{+} \simeq 50-150, \Delta z^{+} \simeq 15-40$ in order to accurately represent the coherent structures in the near-wall region (see Piomelli [11]).

Wall-resolved Large Eddy Simulations of a full-scale vehicle will remain infeasible for many years, but some combination of RANS and LES methods [12] or Detached Eddy Simulation [13] will probably be possible. In the RANS-LES model, LES is used outside the boundary layer, giving a good prediction of large scale structures. The boundary layer is modeled with RANS, which drastically decreases the required near wall resolution. In Detached Eddy Simulation, LES and the RANS model are combined in a single model. This has an effect similar to that of the RANS-LES model, with the main difference that the RANS-LES model is implemented in a zonal way.

The purpose of this paper is to present LES of the flow around simplified bus-like body where the coherent structures near the wall are not resolved but modeled using wall functions based on the 'instantaneous logarithmic low'. The authors are aware of the fact that there is no such thing as 'instantaneous' wall functions. Still, having first node at $y^{+} \simeq 15-30$, it is better to use 'instantaneous' wall functions than to use linear velocity profile.

\section{THE NUMERICAL METHOD}

Calculations are performed with the CALC-BFC [14]. The CALC-BFC code is based on a 3-D finite-volume method for solving the incompressible Navier-Stokes equations employing a collocated grid arrangement. Both convective and viscous fluxes are approximated by central differences of second-order accuracy. Many papers present LES in which dissipative upwind schemes are used for space discretization. This approach increases numerical diffusion, which can lead to a more smooth (unphysical) solution than the correct solution. It is also difficult to distinguish between the influence of the model and the numerics on the results. We have observed some numerical wiggles in front of the model in this work, but they are believed to have a negligible influence on the statistics downstream. A CrankNicolson second-order scheme was used for time integration. The SIMPLEC algorithm is used for the pressure-velocity coupling. The code is parallelized using block decomposition and the message passing systems PVM and MPI [15].

\section{GOVERNING EQUATIONS AND SUBGRID-SCALE MODELING}

In LES, the contribution of the large, energy carrying scales to momentum and energy transfer is computed exactly, and only the effect of the smallest scales of the turbulence is modeled. Decomposition into a large scale component and a small subgrid scale is done by applying a filtering operation:

$$
\bar{f}\left(x_{i}\right)=\int_{\Omega} f\left(x_{i}^{\prime}\right) G\left(x_{i}, x_{i}^{\prime}\right) d x_{i}^{\prime}
$$

where $\Omega$ is the entire flow domain. This filtering operation is applied implicitly with the discretization grid but can also be done explicitly [16]. Top hat filter in real space

$$
G\left(x_{i}, x_{i}^{\prime}\right)= \begin{cases}1 / \Delta, & \text { if }\left|x_{i}-x_{i}^{\prime}\right| \leq \Delta / 2 \\ 0, & \text { otherwise }\end{cases}
$$

where $\Delta$ is the characteristic filter width is used in this work. Filtering the Navier-Stokes and the continuity equations gives the governing equations:

$$
\begin{gathered}
\frac{\partial \bar{u}_{i}}{\partial t}+\frac{\partial}{\partial x_{j}}\left(\bar{u}_{i} \bar{u}_{j}\right)=-\frac{1}{\rho} \frac{\partial \bar{p}}{\partial x_{i}}+\nu \frac{\partial^{2} \bar{u}_{i}}{\partial x_{j} \partial x_{j}}-\frac{\partial \tau_{i j}}{\partial x_{j}} \\
\frac{\partial \bar{u}_{i}}{\partial x_{i}}=0
\end{gathered}
$$

The effect of the small scales appears in the SGS stress tensor, $\tau_{i j}=\overline{u_{i} u_{j}}-\bar{u}_{i} \bar{u}_{j}$, which must be modeled.

The Smagorinsky model is used for modeling the effect of the small scales in this work. It represents the anisotropic part of the SGS stress tensor, $\tau_{i j}$, as:

$$
\tau_{i j}-\frac{1}{3} \delta_{i j} \tau_{k k}=-2 \nu_{s g s} \bar{S}_{i j}
$$

Here

$$
\begin{aligned}
\bar{S}_{i j}=\frac{1}{2}\left(\frac{\partial \bar{u}_{i}}{\partial x_{j}}+\frac{\partial \bar{u}_{j}}{\partial x_{i}}\right) ; \nu_{s g s} & =\left(C_{s} \Delta\right)^{2}|\bar{S}| \\
|\bar{S}| & =\left(2 \bar{S}_{i j} \bar{S}_{i j}\right)^{\frac{1}{2}}
\end{aligned}
$$

The Smagorinsky constant, $C_{s}$, equal to 0.1 is used in this work. 


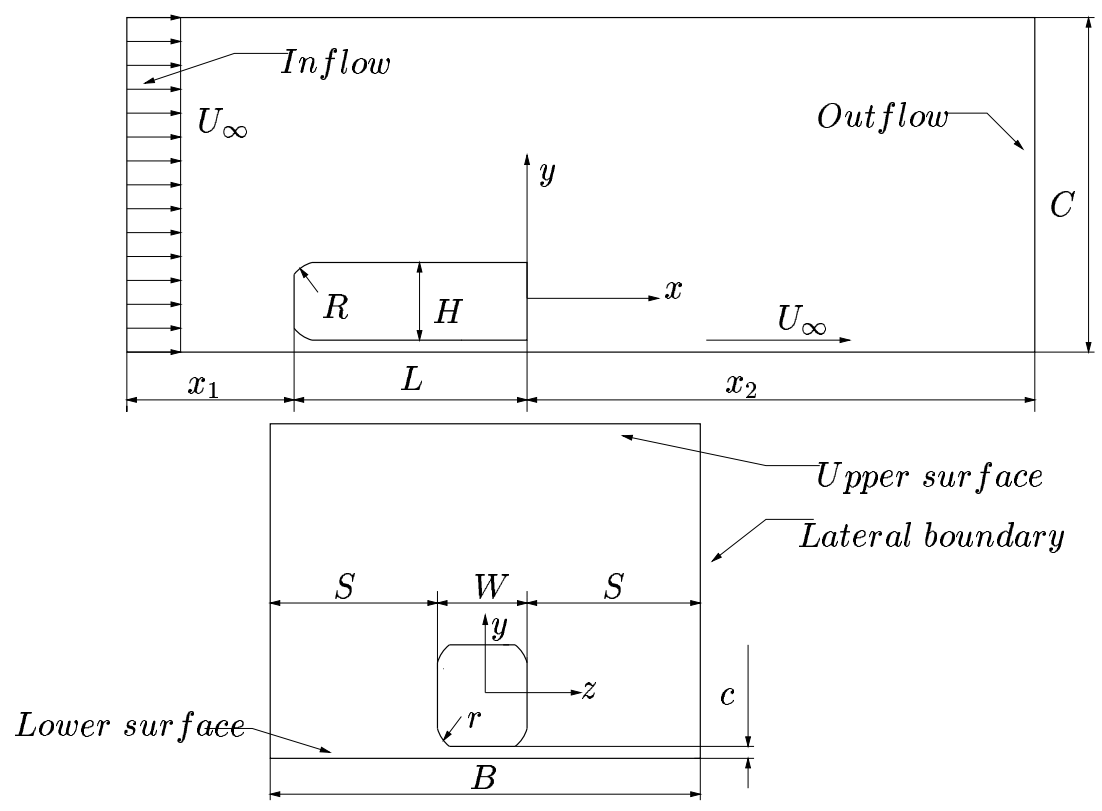

Figure 1: Geometry of the vehicle body and computational domain.

\section{GEOMETRY AND NUMERICAL DETAILS}

The geometry of the computational domain is given in Fig.1. For the simulation, a domain with an upstream length of $x_{1} / H=8$ and a downstream length of $x_{2} / H=16$ was used, while the span-wise width was set at $B=5.92 H$. Similar values for upstream and downstream lengths were found sufficient by Sohankar et al. [17] in Large Eddy Simulation of the flow around a square cylinder. The values of other geometry quantities are $L=0.46 \mathrm{~m}, H=0.125 \mathrm{~m}, W=0.125 \mathrm{~m}$, $S=0.3075 \mathrm{~m}, R=0.019 \mathrm{~m}, r=0.0127 \mathrm{~m}, c=0.01 \mathrm{~m}$ and $C=0.5 \mathrm{~m}$. The ground clearance of $c / H=0.08$ is similar to the clearance ratio of buses. Reynolds number $R e=U_{\infty} H / \nu$ was 210000 based on the incoming mean velocity, $U_{\infty}$, and the vehicle height, $H$. A mesh of 1.8 million nodes was used. The time step was set to 0.0002 seconds, which gave a maximum CFL number of approximately 4.5. The CFL number was smaller than one in $98 \%$ of the cells. The averaging time in the simulation was $t U_{\infty} / H=48$ (30000 time steps). The computational cost using 24 SGI R10000 CPUs was $\simeq 600$ hours (elapsed time).

\section{BOUNDARY CONDITIONS}

Inlet boundary conditions are often obtained by doing a Large Eddy Simulation of a channel flow. The instantaneous velocity profile from this simulation is then used as the inlet boundary condition. In the experiments of Duell and George [1], the inlet mean velocity was uniform within $1 \%$ and the average turbulent intensity was $0.3 \%$. A uniform velocity profile constant in time was thus used as the inlet boundary condition in this work. Convective boundary conditions were applied at the outlet. The convective boundary conditions are of the form $\frac{\partial \bar{u}_{i}}{\partial t}+U_{c} \frac{\partial \bar{u}_{i}}{\partial x_{j}}=0$, with $U_{c}$ being equal to inlet velocity $U_{\infty}$. To simulate the moving ground, the velocity of the lower wall was set equal to $U_{\infty}$. The lateral surfaces were treated as slip surfaces. The wall functions based on the 'instantaneous logarithmic law' are used at all walls.

INSTANTANEOUS WALL FUNCTIONS In this work, we have not resolved the coherent structures in the near wall region owing to high Reynolds number. These coherent structures can be represented if the resolution is $y^{+}<2, \Delta x^{+} \simeq 50-150$ and $\Delta z^{+} \simeq 15-40$ [11]. This requirement implies a computational mesh in the order of $10^{8}$ nodes [18] for the flow case presented in this paper. Such spatial resolution would lead to a smaller time step owing to an increased CFL number, making wall-resolved LES of this flow infeasible. Therefore an approximate wall boundary condition was used in this work. The instantaneous logarithmic law of the form

$$
\bar{u}^{+}=\frac{\ln y^{+}}{0.4}+5.2
$$

is used in the logarithmic region $\left(y^{+} \geq 11.63\right)$. Here, $\bar{u}^{+}=\bar{u} / \bar{u}_{\tau}$ and the friction velocity is defined as $\bar{u}_{\tau}=$ $\left(\tau_{\text {wall }} / \rho\right)^{1 / 2}$. Point $y^{+}=11.63$ is defined as the intersection point between the near wall linear law and the logarithmic law. The linear law $\left(y^{+} \leq 11.63\right)$ is of the form

$$
\bar{u}^{+}=y^{+} \text {. }
$$

The approximate boundary condition when $y^{+} \geq 11.63$ is implemented in the code by adding the artificial viscosity, $\nu_{b c}$, resulting from the approximate wall boundary 
condition (6) to the laminar viscosity on the wall. The friction velocity, $\bar{u}_{\tau}$, is first computed from (6). The wall shear stress is then modeled as

$$
\tau_{\text {wall }} / \rho \equiv\left(\nu \frac{\partial \bar{u}}{\partial y}\right)_{\text {wall }}=\nu_{b c} \frac{\bar{u}}{y}
$$

where $\tau_{\text {wall }}$ is the wall shear stress. The artificial viscosity is now determined from the definition of the friction velocity and Eq. 8 as

$$
\nu_{b c}=\frac{\bar{u}_{\tau}^{2} y}{\bar{u}}=\frac{\bar{u}_{\tau} y}{\bar{u}^{+}}
$$

where $\bar{u}^{+}$is obtained from (6). The spatial resolution expressed in the wall units was $y^{+} \simeq 15-30, \Delta x^{+} \simeq$ $100-600$ and $\Delta z^{+} \simeq 100-300$, as recommended by Piomelli [11].

\section{SOME EXPERIMENTAL DETAILS}

This section gives some details from the experiments of Duell and George [1] for the purpose of comparison with LES. The cross section of the tunnel test section and the ground clearance are the same as in the LES simulation. The location of the model front side relative to the inlet was $0.564 \mathrm{~m}$. The distance from the test section exit to the back wall perpendicular to the flow was $1.854 \mathrm{~m}$. This is the back wall where Duell and George mounted the deflector to reduce low frequency pulsations [19]. A moving ground belt and boundary layer scoop were used to simulate the floor boundary condition and to minimize boundary layer effects. Velocity measurements were done using hot wire anemometry. The velocity reported in Ref. [1] is the mean effective cooling velocity, $\bar{V}_{e f f}$, defined as $\bar{V}_{e f f}=\left(\bar{U}^{2}+\bar{V}^{2}\right)^{1 / 2}$ where $\bar{U}$ and $\bar{V}$ are the mean velocity components in the $x$ and $y$ directions (see Fig. 1). For comparison with the experiments, $\bar{V}_{\text {eff } f}$ was computed in LES as $\bar{V}_{\text {eff }}=\left(\langle\bar{u}\rangle_{t}{ }^{2}+\langle\bar{v}\rangle_{t}{ }^{2}\right)^{1 / 2}$. Here, $\langle\bar{u}\rangle_{t}$ and $\langle\bar{v}\rangle_{t}$ are timeaveraged resolved velocity components in the $x$ and $y$ directions obtained from LES.

\section{RESULTS}

STATISTICS OF THE MEAN FLOW AND GLOBAL QUANTITIES Time-averaged velocities are computed and compared with the experiments. The results are presented in Fig. 2. As can be seen, the agreement with LES results is good in the recirculation region $(x / H=0.32, x / H=0.98)$, while the velocity profile is under-predicted behind the recirculation $(x / H=1.63)$. The location of the free stagnation point was found to be below the center of the base of the model at $x=1.6 H$, $y=-0.17 H$ (see Fig. 3). In the experiments of Duell and George [1], the free stagnation point was assumed to be at $y=0$. They plotted $\bar{V}_{e f f} / U_{\infty}$ along the $x$-axis
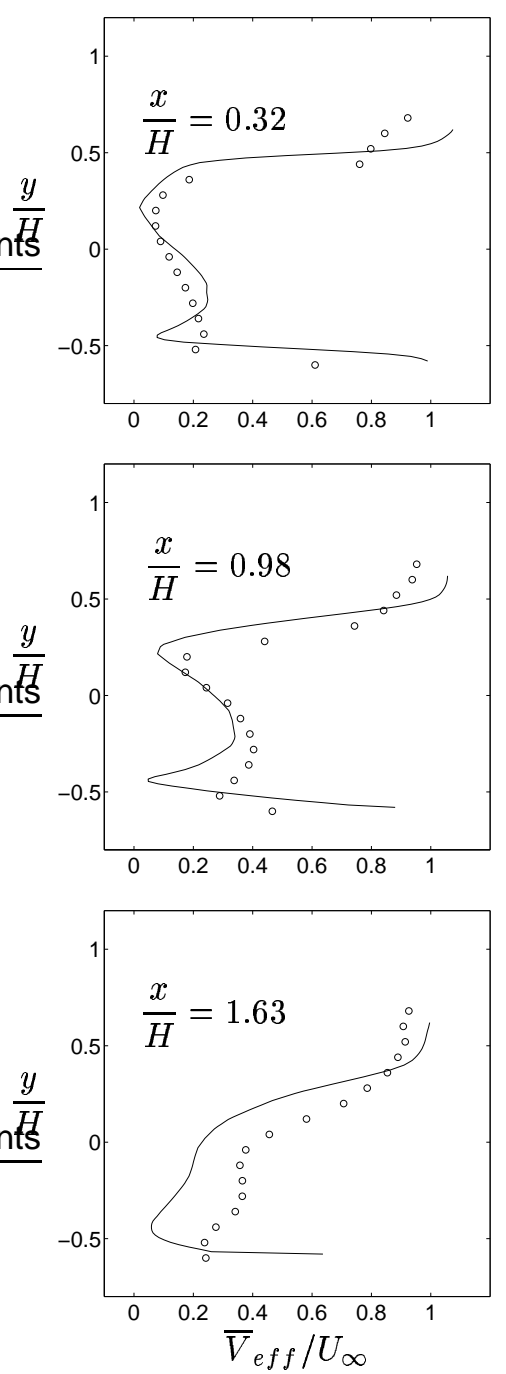

Figure 2: Time-averaged velocity profiles at three downstream locations at $z=0$. LES (solid line); experiment (symbols).

and used the local minimum as indication of the free stagnation point. The distribution of the mean velocity components, $\langle\bar{u}\rangle_{t} / U_{\infty}$ and $\bar{V}_{e f f} / U_{\infty}$, at $y=0$ obtained in our LES are plotted in Fig 4. The recirculation length was found to be $X_{r}=1.36 \mathrm{H}$ using the local minimum of $\bar{V}_{\text {eff }} / U_{\infty}$ and $X_{r}=1.44 \mathrm{H}$ using the intersection of $\langle\bar{u}\rangle_{t} / U_{\infty}$ with the $x$-axis. This is larger than $X_{r}=1.1 H$, measured in the experiment of Duell and George [1].

Time-averaged velocity vectors in plane $z=0$ are shown in Fig. 3. It can be seen that the lower entrapped vortex (center at $x=0.7 H, y=-0.43 H$ ) is much smaller than the upper vortex (center at $x=0.33 H$, $y=0.24 H$ ). This owing to small ground clearance, which minimizes the amount of fluid that can be entrained by the lower trapped vortex [1]. Time-averaged velocity vectors projected onto plane $y=0$ are shown in 


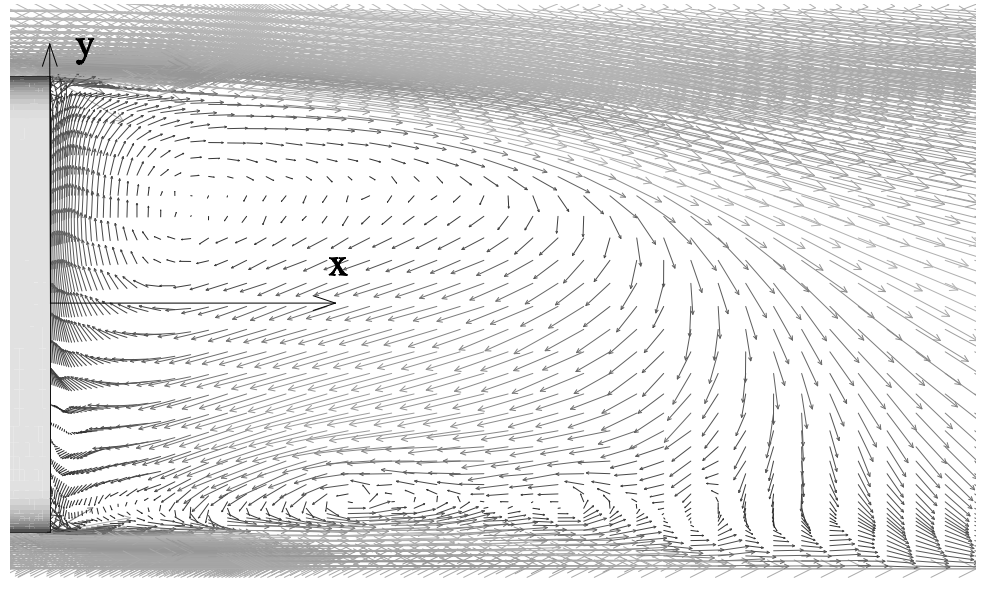

Figure 3: Time-averaged velocity vectors in the symmetry plane $(z=0)$.

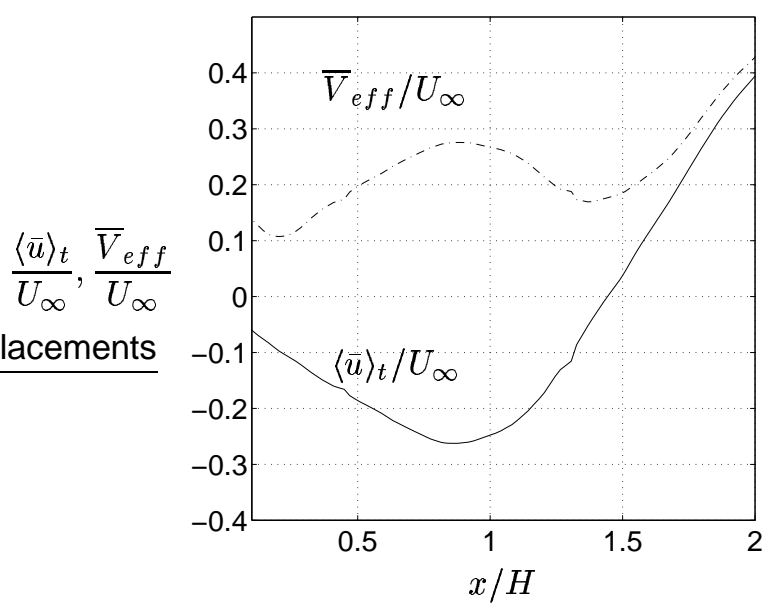

Figure 4: Distribution of the mean velocity component $\langle\bar{u}\rangle_{t} / U_{\infty}$ (solid line) and the mean velocity $\bar{V}_{\text {eff }} / U_{\infty}$ (dashed-dotted line) along the $x$-axis at $y=0, z=0$.

Fig. 5. Both foci at $x=0.91 H, z= \pm 0.35 H$ are clearly visible in this figure. The saddle point at $x=1.54 \mathrm{H}$, $z=0$, indicating the free stagnation point, is also visible. As can be seen in Fig. 5, the two recirculation regions downstream of the body are symmetric, which indicates that the number of samples and the averaging time, 30000 time steps $\left(t U_{\infty} / H=48\right)$, are sufficient.

Comparison of the base pressure coefficient $C_{p_{\text {base }}}=$ $\left(p-p_{\infty}\right) /\left(0.5 \rho U_{\infty}^{2}\right)$ between the experiment and LES is shown in Fig. 6 . It can be seen that the shape of the profile is the same, whereas the level of $C_{p_{\text {base }}}$ is overpredicted. The integrated value of $C_{p_{\text {base }}}$ over the base surface in LES was $\bar{C}_{p_{\text {base }}}=-0.23$ while, in the experiment, the value of $\bar{C}_{p_{\text {base }}}=-0.286$ was measured.

COHERENT STRUCTURES A ring vortex behind the body is shown instantaneously in Fig. 7 and in the mean

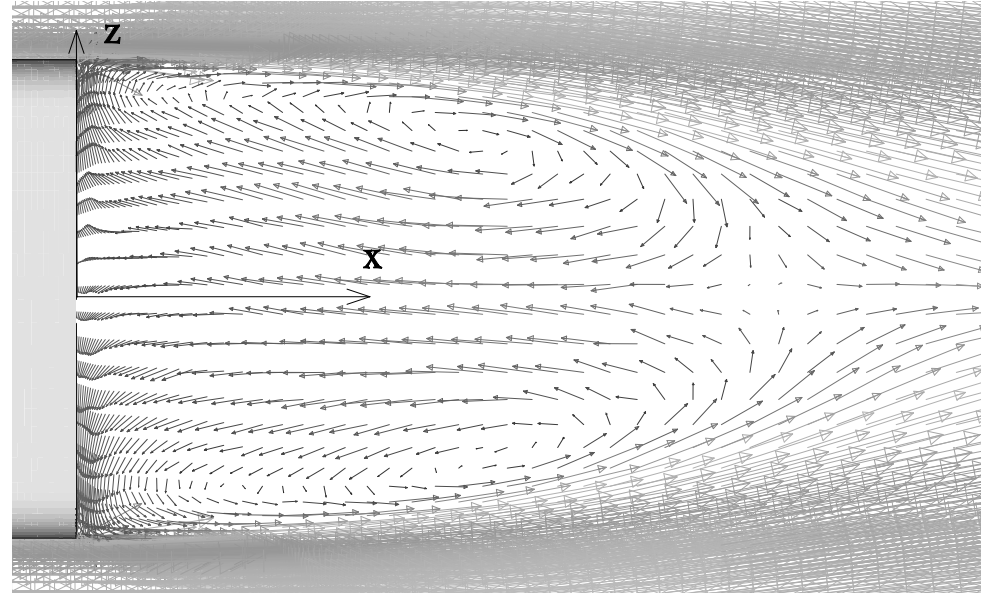

Figure 5: Time-averaged velocity vectors in plane $y=0$.

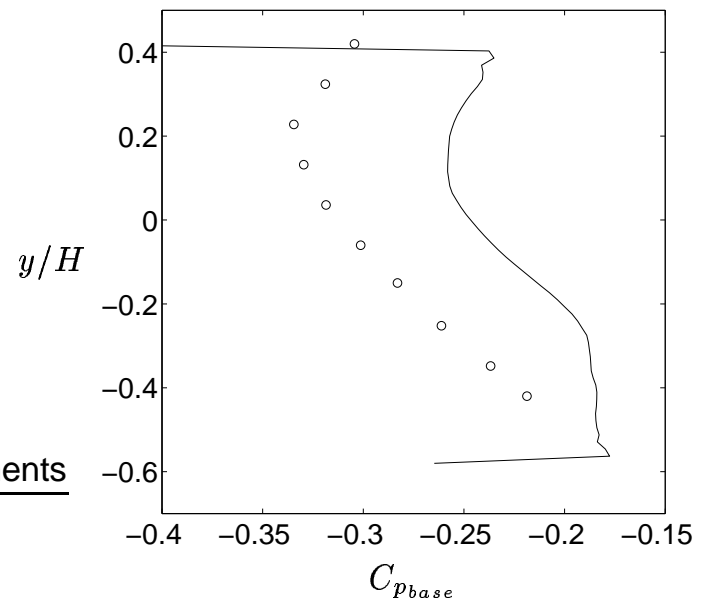

Figure 6: Time-averaged base pressure distribution along the $y$-axis. Experimental data (symbols); LES (solid line).

in Fig. 8. Interaction between the upper and the lower trapped vortices that forms the ring vortex can be followed in Fig. 7. It was found in the experiments that this is a periodic process that can be measured at the model base. The Strouhal number of this pumping in LES is $S t=0.073$ (see Fig. 9) as compared with the experimental $S t=0.069$. In the mean, these vortices build a ring vortex visualized in Fig. 8. The surface of the lowest pressure shows only half of the ring (see Fig. 8d), which grows to full size with slightly higher pressure (see Fig. 8a).

Figure 10 shows the instantaneous second invariant of the velocity gradient, $Q=0.01$, colored with the velocity magnitude, for two different instants. $Q$ is defined as

$$
Q=-\frac{1}{2} \frac{\partial \bar{u}_{i}}{\partial x_{j}} \frac{\partial \bar{u}_{j}}{\partial x_{i}}=-\frac{1}{2}\left(\bar{S}_{i j} \bar{S}_{i j}-\bar{\Omega}_{i j} \bar{\Omega}_{i j}\right)
$$

where $\bar{\Omega}_{i j}$ is the anti-symmetric part of the resolved velocity gradient tensor. This technique divides regions of 


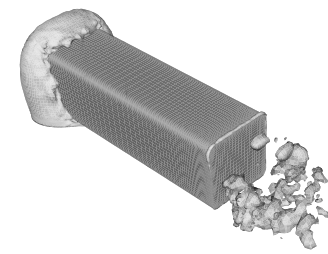

a)
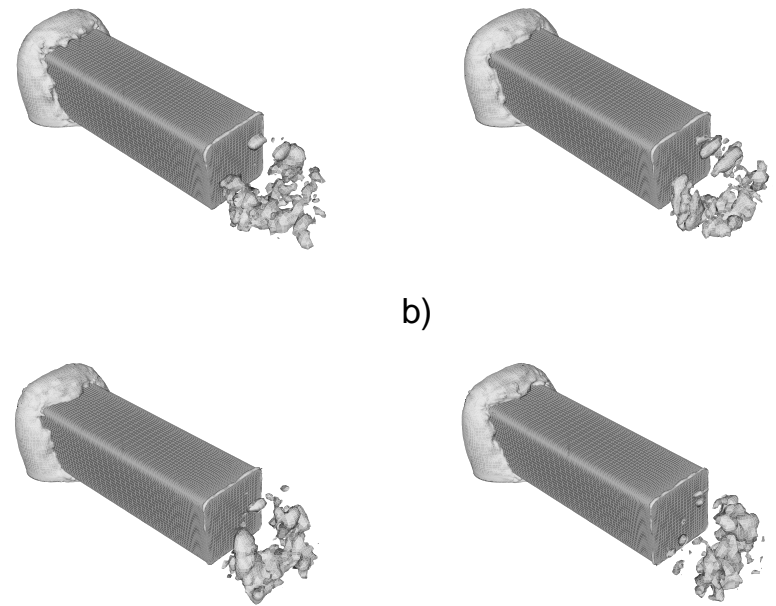

c)

b)

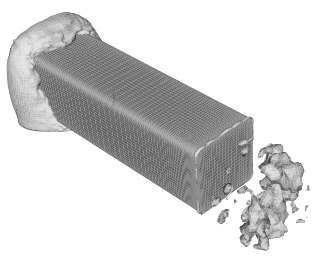

d)

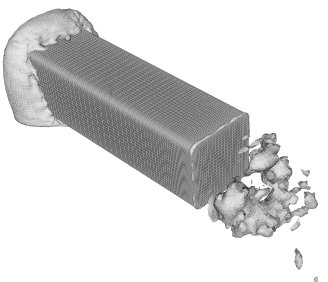

e)

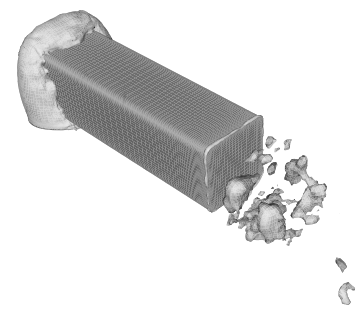

f)

Figure 7: Instantaneous isosurface of low value negative pressure, $p=-0.109$, at six different times. View of the downstream face of the vehicle.
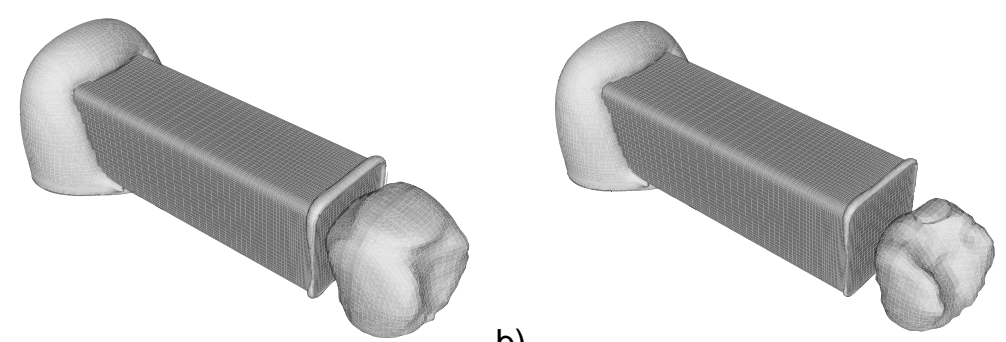

b)

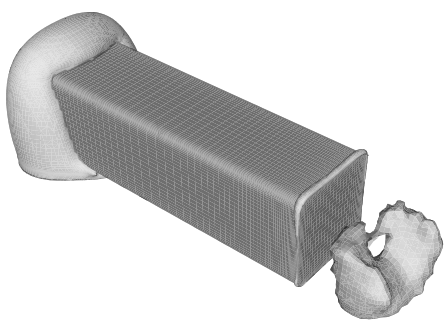

d)

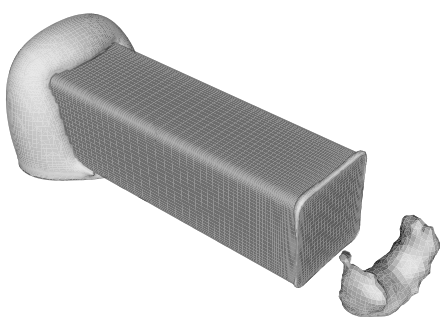

Figure 8: Isosurface of a time-averaged negative pressure: a) $p=-0.1045$; b) $p=-0.1057$; c) $p=$ -0.1060 ; d) $p=-0.1063$. View of the downstream face of the vehicle.

high and low pressures and thereby visualizes vortices. The vortex pairing mechanism can be studied, following vortices from the separation at the base downstream of the body.
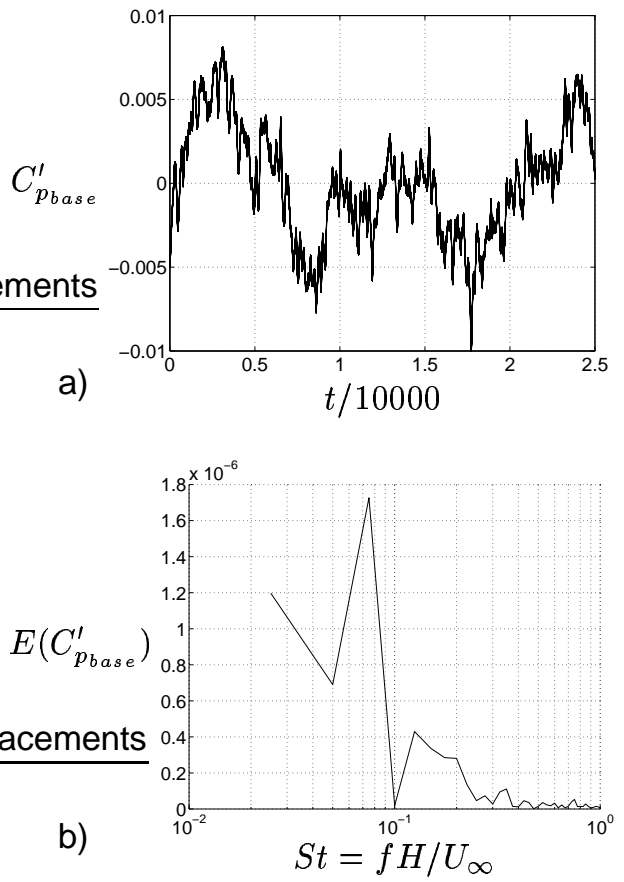

Figure 9: a) Time history of $C_{p_{\text {base }}}^{\prime}$. b) The power density spectrum of the $C_{p_{\text {base }}}^{\prime}$ signal.
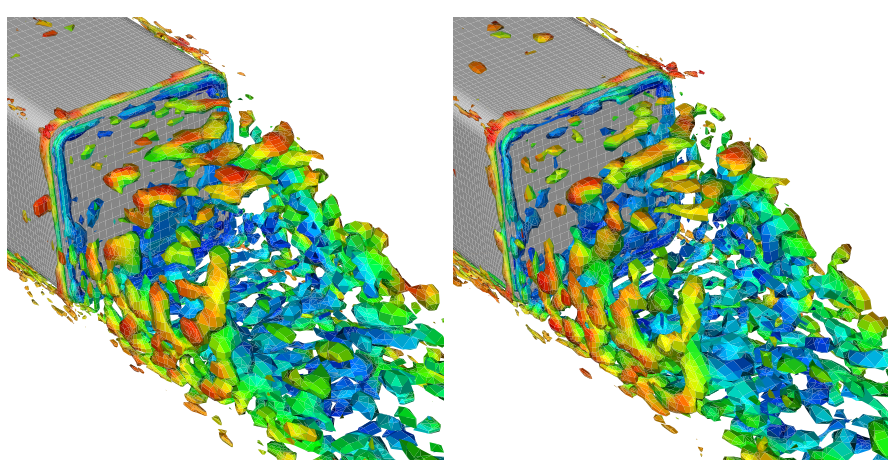

Figure 10: Instantaneous second invariant of the velocity gradient, $Q=0.01$, colored with the velocity magnitude. The time difference between two pictures is $t U_{\infty} / H=0.32$. View of the downstream face of the vehicle.

Downstream of the body, the three-dimensional boundary layers on the upper and lower sides form the shear layers shown in Fig. 11. This shear layer separates at the sharp edge of the base of the model. Figure 12 shows the shear layers generated from the sides of the model. Here as well the separation is defined by the sharp edges of the base of the model. The vortex shedding in the span-wise direction can be followed in Fig. 12. Some numerical wiggles are present upstream of the body owing to the centered difference scheme. These oscillations are present in front of the model only.

Figure 13 plots the time-averaged vorticity component, $\omega_{z}$. Two reverse flow regions resulting from the 
a)

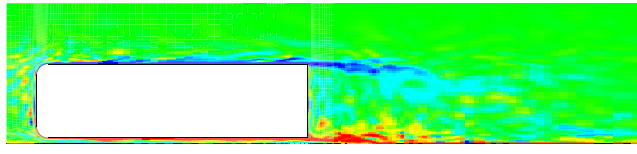

b)

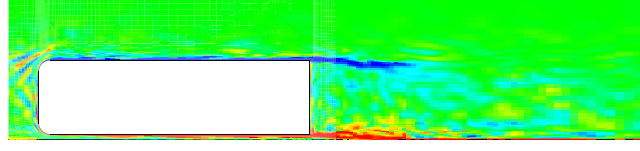

c)

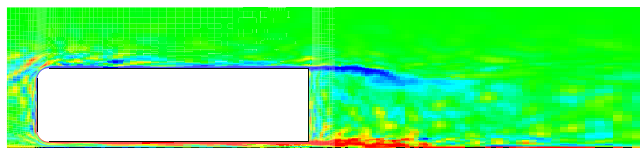

Figure 11: Instantaneous vorticity component $\omega_{z}$ at $z=$ 0. a) $t U_{\infty} / H=0$; b) $t U_{\infty} / H=1.76$; c) $t U_{\infty} / H=3.52$.

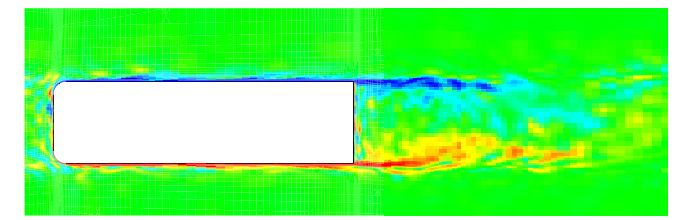

a)

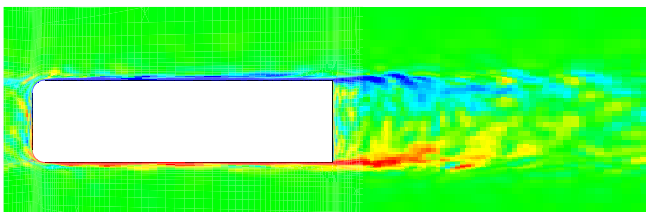

b)

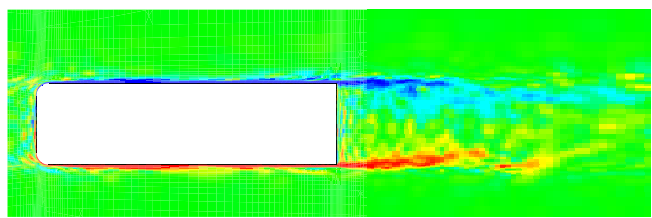

c)

Figure 12: Instantaneous vorticity component $\omega_{y}$ at $y=$ 0. a) $t U_{\infty} / H=0$; b) $t U_{\infty} / H=1.76$; c) $t U_{\infty} / H=3.52$.

shear layers on the upper and lower sides of the body are visible. The same pair of recirculating flow regions can be seen in Fig. 14, which shows the mean vorticity component, $\omega_{y}$. Following the swirling motion of the stream-ribbons makes it possible to identify the core of the ring vortex resulting from the interaction of these recirculating regions in Fig. 15

Figure 16 shows the time-averaged vorticity component $\omega_{x}$ at five different positions. The shape of the two main vortices in the wake can be followed in this figure. A pair of trailing vortices close to the lower corners of the body is also visible in this figure. The formation of the trailing vortices is also visualized using timeaveraged streamlines in Fig. 17 and velocity vectors in Fig. 18. This process is studied in Fig. 19. It was found that the mean trailing vortices form as a result of several instantaneous vortices (see Fig. 19). a)

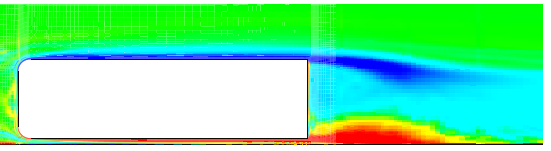

b)

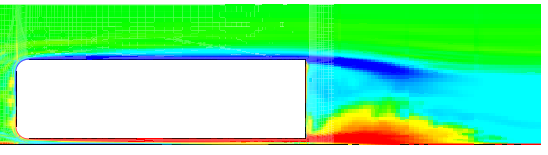

c)

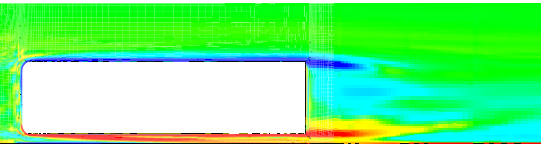

Figure 13: Time-averaged vorticity component $\omega_{z}$. a) $z=0$; b) $z=0.24 \mathrm{H}$; c) $z=0.48 \mathrm{H}$. Blue is clockwise rotation (negative $\omega_{z}$ ) and red is counter-clockwise rotation (positive $\omega_{z}$ ).

a)

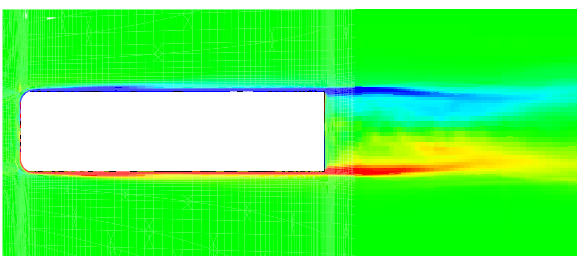

b)

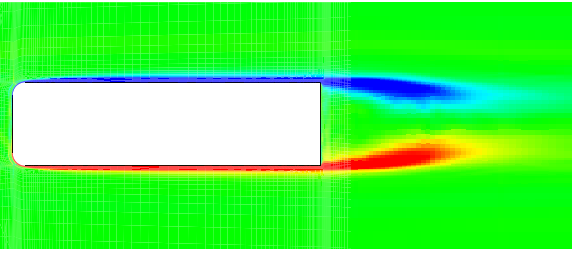

c)

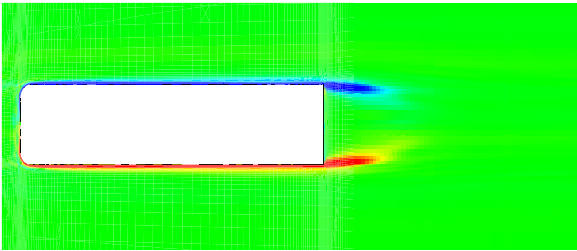

Figure 14: Time-averaged vorticity component $\omega_{y}$. a) $y=-0.38 \mathrm{H}$; b) $y=0.02 \mathrm{H}$; c) $y=0.46 \mathrm{H}$. Blue is clockwise rotation (negative $\omega_{y}$ ) and red is counterclockwise rotation (positive $\omega_{y}$ ).

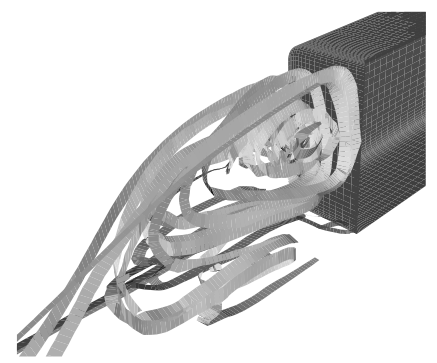

Figure 15: Time-averaged stream-ribbons in the near wake. Only half of the near wake is shown, and the cut is made at plane $z=0$. View from the symmetry plane.

UNSTEADY VELOCITY SIGNALS IN THE WAKE The velocity signals for two points in the near wake 
a)

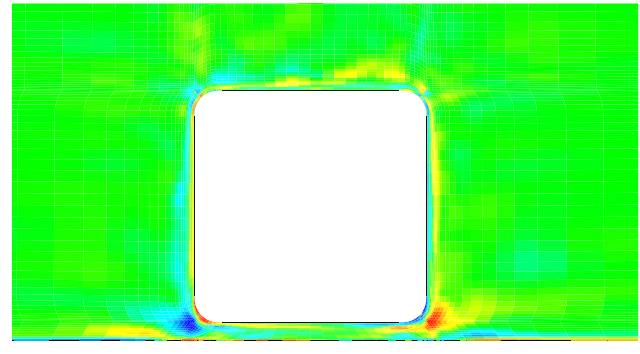

b)

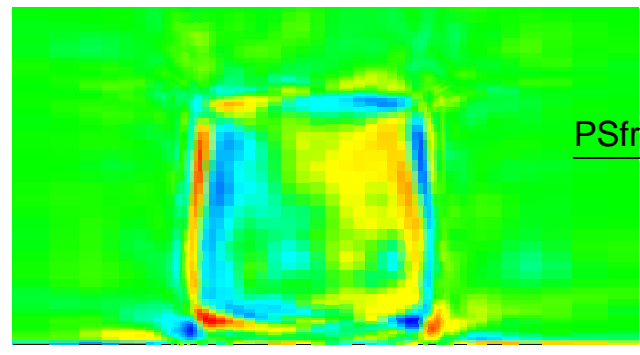

c)

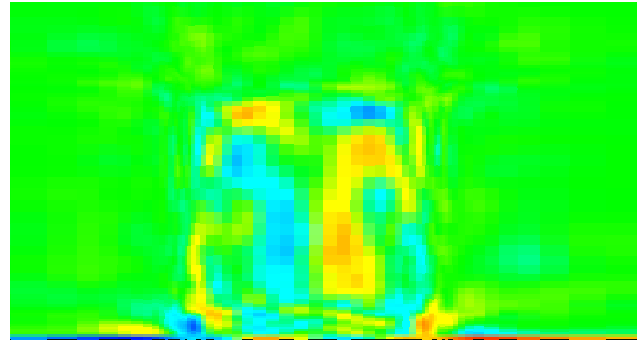

d)

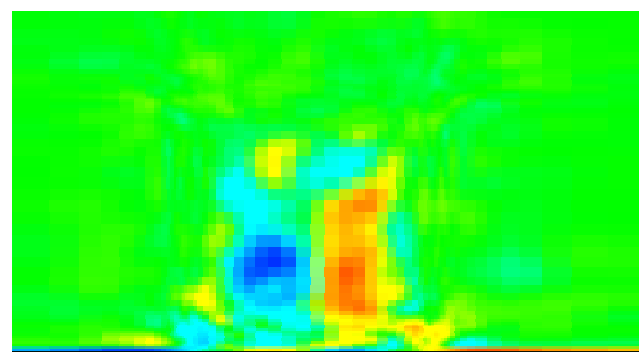

e)

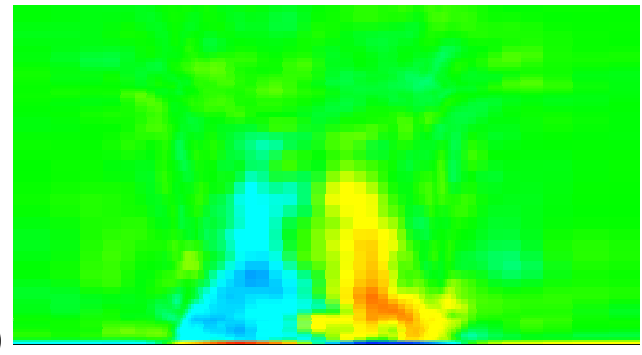

Figure 16: Time-averaged vorticity component $\omega_{x}$. a) $x=0$; b) $x=0.48 H$; c) $x=0.98 H$; d) $x=1.76 H$; e) $x=2.72 H$. Blue is clockwise rotation (negative $\omega_{x}$ ) and red is counter-clockwise rotation (positive $\omega_{x}$ ).

were sampled and their fluctuations are presented in Figs. 20a and 21a. The locations of the selected points are in the shear layer of the recirculation region and in the wake region close to the free stagnation point. The same points were chosen for the velocity signal sampling in the experiments of Duell and George [1]. The power density spectrum at the two points resulting from LES is presented in Figs. 20b and 21b. Four distinct pe-

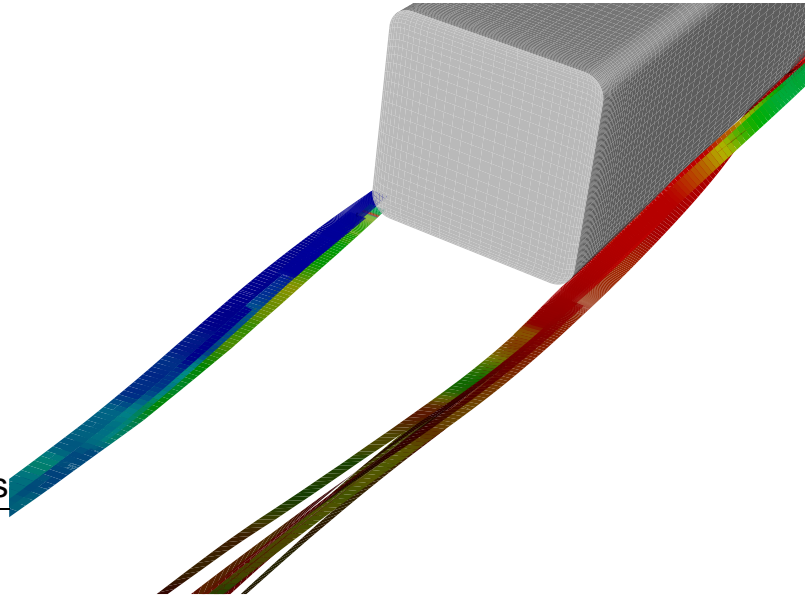

Figure 17: Time-averaged vortices generated the in interaction of the shear layers on the vehicle and the flow from below the body. They are coloured with vorticity component $\omega_{x}$. Blue is clockwise rotation (negative $\omega_{x}$ ) and red is counter-clockwise rotation (positive $\omega_{x}$ ). View of the downstream face of the vehicle.

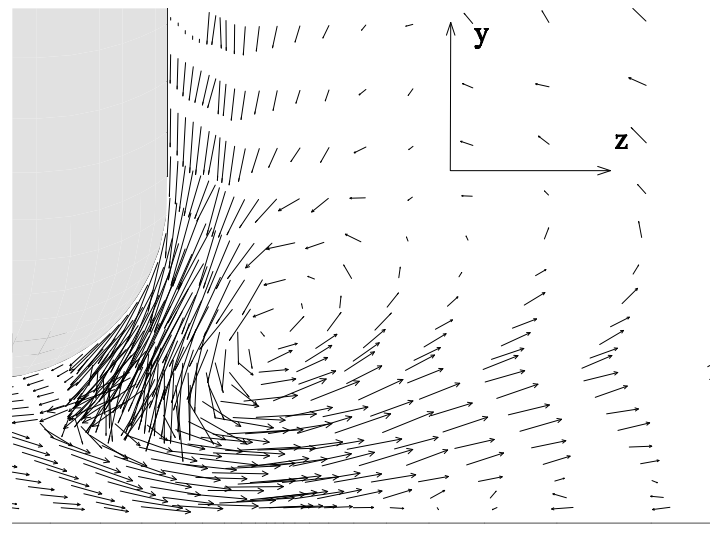

Figure 18: Time-averaged velocity vectors at $x=$ $-0.48 H$

aks are measured near the separation point in Fig. 20b. They correspond to non-dimensional frequencies $S t=$ $0.032, S t=0.21, S t=0.29$ and $S t=0.88$. In the experiment, Duell and George [1] measured $S t=1.157$ at this position. The power density spectrum at the second point close to the free stagnation point has distinct peaks at $S t=0.11, S t=0.26, S t=0.51, S t=0.73$ and $S t=1$ (see Fig. 21b). Only one distinct peak at $34 \mathrm{~Hz}$ corresponding to $S t=0.155$ was observed in the experiment [1]. Duell and George concluded that this peak indicates the vortex pairing close to the free stagnation point. The largest peak, $S t=0.11$, in the LES simulation is fairly close to the experimental value, $S t=0.155$.

SURFACE PRESSURE The surface pressure gives the dominant contribution to the drag and was thus stu- 


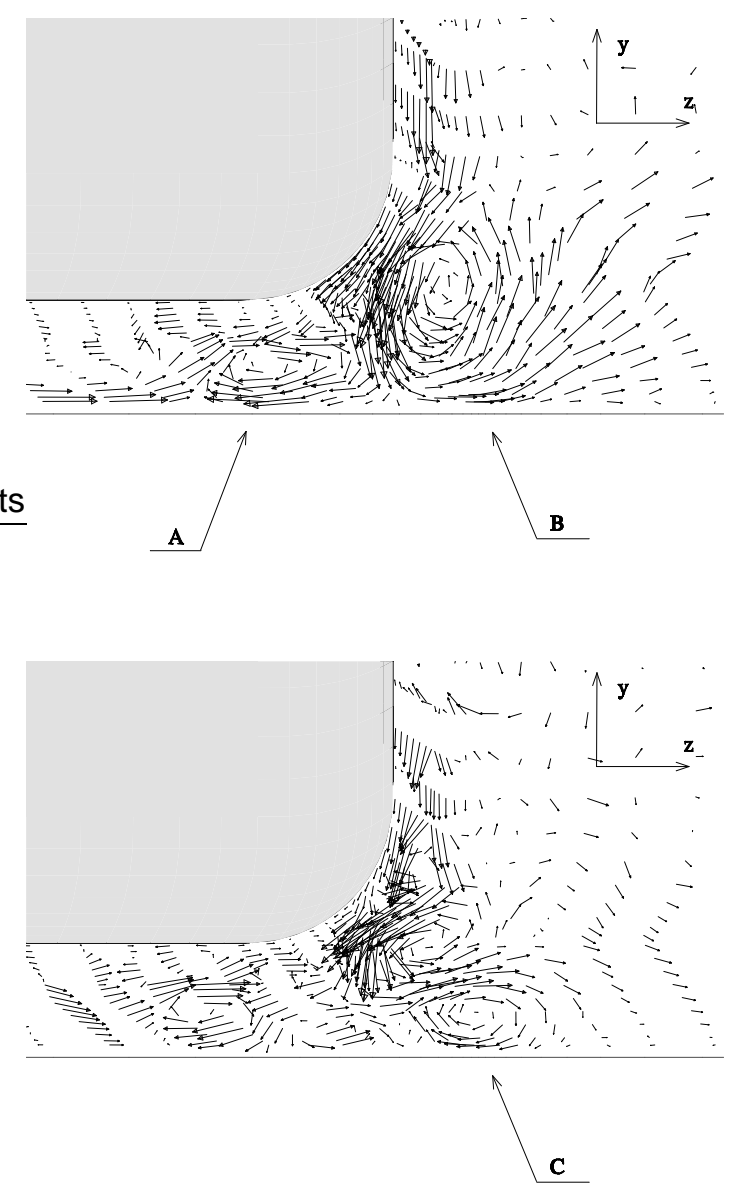

Figure 19: Instantaneous velocity vectors at $x=$ $-0.48 \mathrm{H}$. Vortices (A, B and C). The time difference between two pictures is $t U_{\infty} / H=0.47$.

died in Figs. 22 and 23 . It was found that the variation in the instantaneous surface pressure was larger in the span-wise direction than from the upper to the lower part of the base. The mean pressure is higher on the lower part of the base of the model than on the upper one (see Figs. 6 and 23). It has a suction peak in the upper half of the base, which was also observed in the experiment [20]. A positive pressure peak is found in the lower half of the base (see Fig. 23), which is not seen in the experiment.

CONCLUSIONS Large Eddy Simulation of the flow around a vehicle body was done, and the results are compared with the experiments of Duell and George [1]. The Reynolds number of 210000 based on the incoming mean velocity and the model height makes this simulation extremely challenging. It is shown that this simulation can be carried out using the approximate wall boundary conditions based on the 'instantaneous' logarithmic low. Time-averaged velocities and some global quantities are determined and compared with experi-
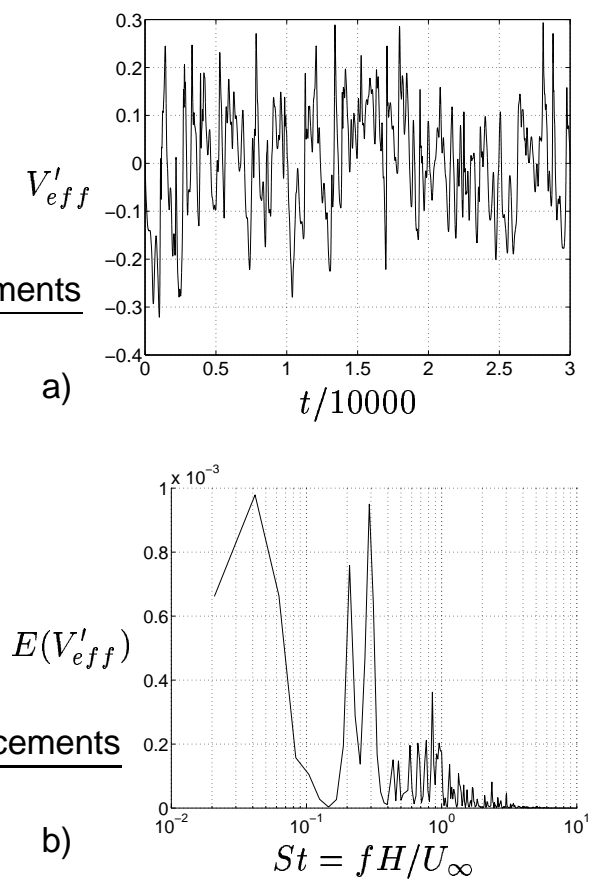

Figure 20: a) Time history of $V_{e f f}^{\prime}=V_{e f f}-\bar{V}_{e f f}$. b) The power density spectrum of $V_{e f f}^{\prime}$ signal. $x=0.1463 H$, $y=0.5 H, z=0$
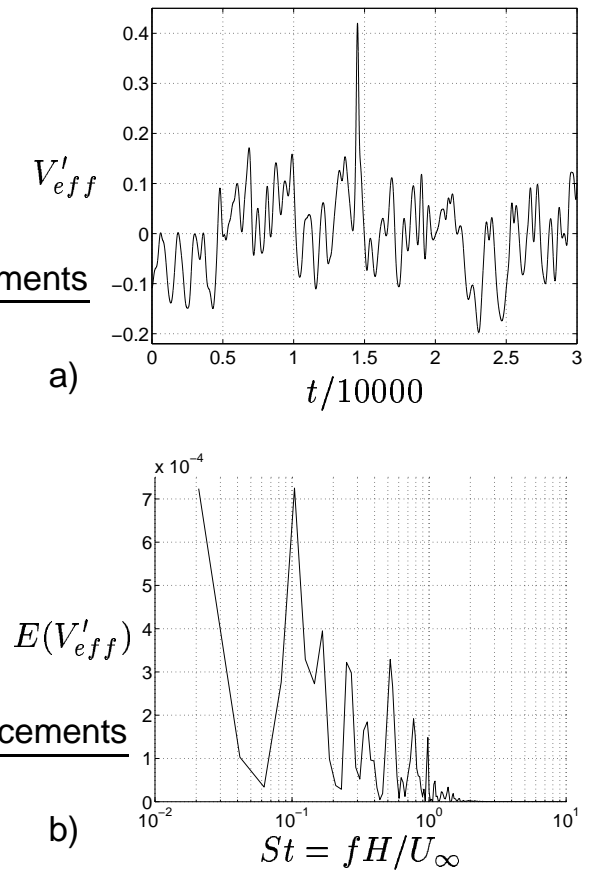

Figure 21: a) Time history of $V_{e f f}^{\prime}=V_{e f f}-\bar{V}_{e f f}$. b) The power density spectrum of $V_{\text {eff }}^{\prime}$ signal. $x=0.9526 \mathrm{H}$, $y=0, z=0$

ments. Although the computed velocity profiles agree fairly well with the experiment, the recirculation length was found to be over-predicted by some $24 \%$. The base pressure coefficient, $C_{p_{\text {base }}}$, from LES exhibits the same shape as the experimental one, although the integrated value, $\bar{C}_{p_{\text {base }}}$, is over-predicted. The interac- 
a)
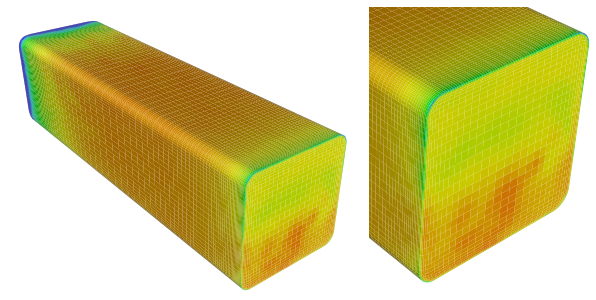

b)
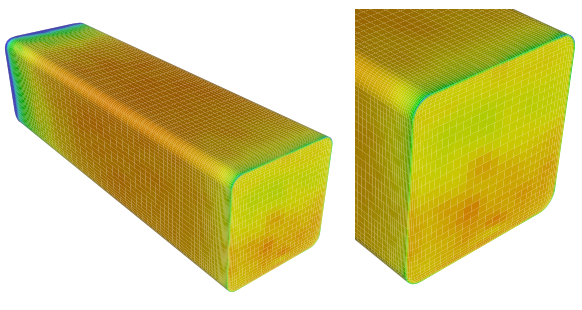

c)
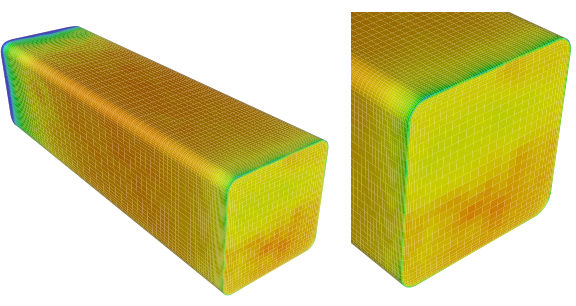

Figure 22: Instantaneous surface pressure. $t U_{\infty} / H=0$; b) $t U_{\infty} / H=1.76$; c) $t U_{\infty} / H=3.52$. Red is high pressure and blue is low pressure. View of the downstream face of the vehicle.

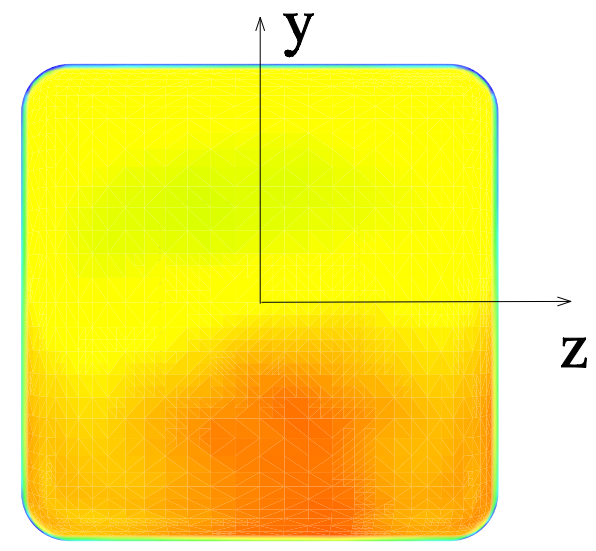

Figure 23: Time-averaged surface pressure at the base of the model. Red is high pressure and blue is low pressure.

tion between upper and lower trapped vortices in the near wake was studied, and the pumping frequency was measured as $S t=0.073$. This frequency is very close to the experimental value of $S t=0.069$. It was found that the coherent structures from this process result in a ring vortex in the near wake. Other coherent structures were observed, such as trailing vortices generated in the shear layer near the lower corners of the side of the body. The velocity signals were sampled for two positions, and the power density spectra are compared with the experiment. The vortex pairing close to the free stagnation point was measured at a frequency of $S t=0.11$ compared to the experimental $S t=0.155$. From this we conclude that the LES, with fairly simple approximate wall boundary conditions, gives accurate results. These results encourage us to proceed with more accurate modeling of the flow using a hybrid LESRANS model.

ACKNOWLEDGMENTS This work was supported by NUTEK and the Volvo Car Corporation. Computer time on the SGI ORIGIN 2000 machines, provided by UNICC at Chalmers, is gratefully acknowledged. The authors thank Dr. E. G. Duell for providing data from his experimental measurements.

\section{REFERENCES}

[1] E. G. Duell and A. R. George. Experimental study of a ground vehicle body unsteady near wake. SAE Paper 1999-01-0812, 1999.

[2] S. Wallin and A. Johansson. An explicit algebraic reynolds stress model for incompressible and compressible turbulent flows. Journal of Fluid Mechanics, 403:89-132, 2000.

[3] T.J. Craft, B.E. Launder, and K. Suga. A non-linear eddy viscosity model including sensitivity to stress anisotropy. In 10th Symp. Turbulent Shear Flows, The Pennsylvania State University, USA, August 1995.

[4] S. Perzon. Private communication. Volvo Car Corporation, 2000.

[5] S. Perzon and L. Davidson. On transient modeling of the flow around vehicles using the reynolds equation. In Wen X.-B. Hu W. Jia F.-P., editor, Int. conference on applied computational fluid dynamics, pages 720-727, Beijing, China, 2000.

[6] W. Rodi, J.H. Ferziger, M. Breuer, and M. Pourquié. Status of large-eddy simulations: Results of a workshop. J. Fluids Engineering, pages 248-262, 1997.

[7] S. Krajnović and L. Davidson. Large eddy simulation of the flow around a three-dimensional bluff body. In 39th AIAA Aerospace Sciences Meeting and Exhibit, AIAA paper 2001-0432, Reno, Nevada, USA, 2001.

[8] S. Krajnović. Large eddy simulation of the flow around a three-dimensional bluff body. Thesis for Licentiate of Engineering 00/1, Dept. of Thermo and Fluid Dynamics, Chalmers University of Technology, Gothenburg, Sweden, January 2000. 
[9] A. Sohankar, L. Davidson, and C. Norberg. Large eddy simulation of flow past a square cylinder: Comparison of different subgrid scale models. ASME: Journal of Fluids Engineering, 122(1):3947, 2000.

[10] A. Sohankar, L. Davidson, and C. Norberg. Erratum. ASME: Journal of Fluids Engineering, 122(3):643, 2000.

[11] U. Piomelli and J.R. Chasnov. Large-eddy simulations: Theory and applications. In D. Henningson, M. Hallbaeck, H. Alfreddson, and A. Johansson, editors, Transition and Turbulence Modelling, pages 269-336, Dordrecht, 1996. Kluwer Academic Publishers.

[12] L. Davidson. LES-RANS of channel flow. Report 00/2, Dept. of Thermo and Fluid Dynamics, Chalmers University of Technology, Göteborg, Sweden, 2000.

[13] M. Shur, P. R. Spalart, M. Strelets, and A. Travin. Detached-eddy simulation of an airfoil at high angle of attack. 1998.

[14] L. Davidson and B. Farhanieh. CALC-BFC: A finite-volume code employing collocated variable arrangement and cartesian velocity components for computation of fluid flow and heat transfer in complex three-dimensional geometries. Rept. 92/4, Dept. of Thermo and Fluid Dynamics, Chalmers University of Technology, Gothenburg, 1992.

[15] H. Nilsson and L. Davidson. CALC-PVM: A parallel SIMPLEC multiblock solver for turbulent flow in complex domains. Int.rep. 98/12, Dept. of Thermo and Fluid Dynamics, Chalmers University of Technology, Gothenburg, 1998.

[16] T. S. Lund. On the use of discrete filters for large eddy simulation. In Annual Research Briefs 1997, pages 83-95, Center for Turbulent Research, Stanford Univ./NASA Ames Reseach Center, 1997.

[17] A. Sohankar. Numerical Investigation of Vortex Shedding Around Square Cylinders at Low Reynolds Number. PhD thesis, Dept. of Thermo and Fluid Dynamics, Chalmers University of Technology, Gothenburg, 1998.

[18] P. R. Spalart. Private communication. Boeing Commercial Airplanes, 2000.

[19] E. G. Duell. Private communication. Svendrup Technology, Inc, 2000.

[20] E. G. Duell. Experimental investigation of unsteady near wakes of ground vehicle bodies. PhD thesis, Cornell University, 1994. 